Erratum

\title{
Erratum to "Water Management Practices Affect Arsenic and Cadmium Accumulation in Rice Grains"
}

\author{
Liming Sun, Manman Zheng, Hongyan Liu, Shaobing Peng, \\ Jianliang Huang, Kehui Cui, and Lixiao Nie
}

National Key Laboratory of Crop Genetic Improvement, MOA Key Laboratory of Crop Ecophysiology and Farming System in the Middle Reaches of the Yangtze River, College of Plant Science and Technology, Huazhong Agricultural University, Wuhan, Hubei 430070, China

Correspondence should be addressed to Lixiao Nie; nielixiao@mail.hzau.edu.cn

Received 11 November 2014; Accepted 17 November 2014; Published 27 November 2014

Copyright (C) 2014 Liming Sun et al. This is an open access article distributed under the Creative Commons Attribution License, which permits unrestricted use, distribution, and reproduction in any medium, provided the original work is properly cited.

In the paper titled "Water management practices affect arsenic and cadmium accumulation in rice grains," the "Cd and As concentration in grains in a field experiment" data of Figure 2 in page 4 appeared incorrectly in this paper. For that particular bar chart, the authors accidentally calculated the " $\mathrm{Cd}$ and As concentration in milled grains" as " $\mathrm{Cd}$ and As concentration in grains." So the grain $\mathrm{Cd}$ and As concentrations were reanalyzed. And the results showed that the original data were quite different from the reanalyzed data. The trends of $\mathrm{Cd}$ and As concentration in rice grains among various water management practices are not affected by this. Please see the corrected Figure 2 . 


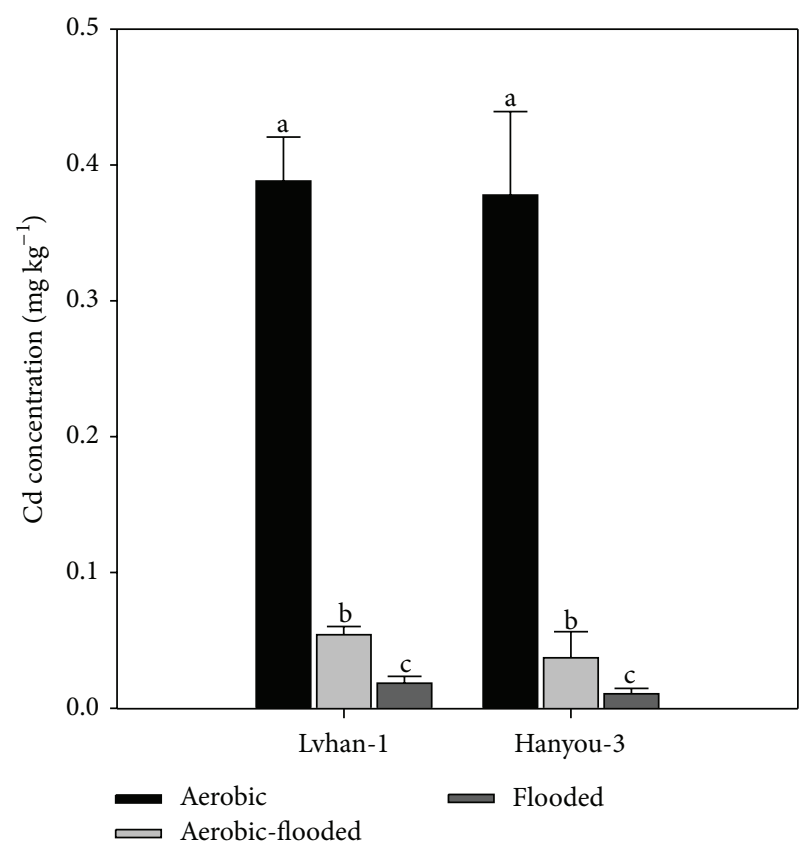

(a)

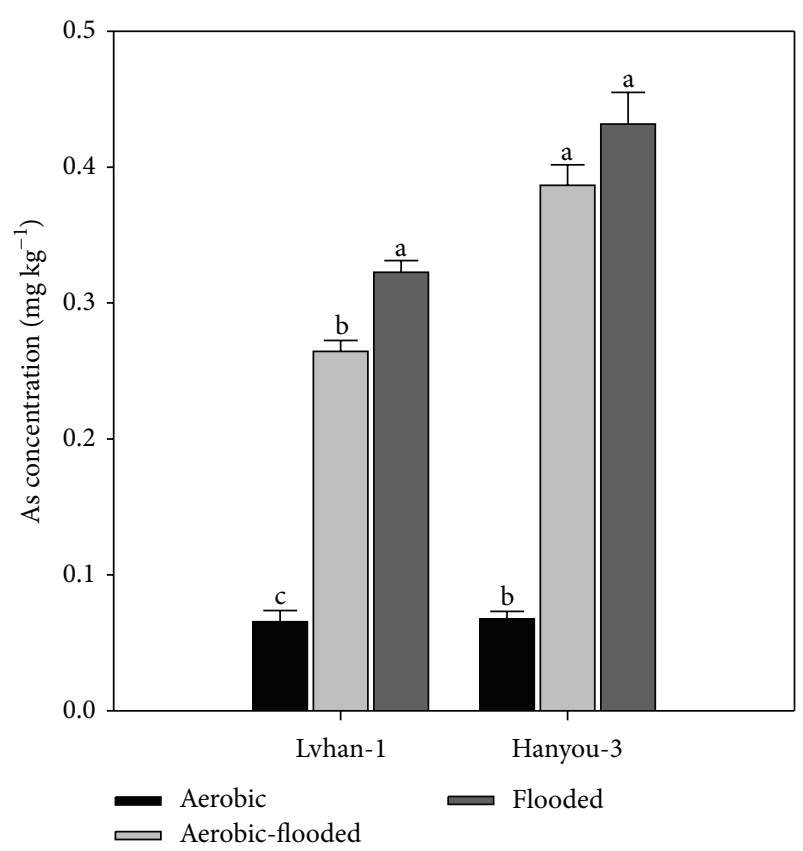

(b)

Figure 2: The Cd and As concentration in grains of Lvhan-1 and Hanyou-3 grown under three water management treatments, aerobic, flooded, and aerobic-flooded cultivation in a field experiment. Error bars represent standard error of mean (SE). 


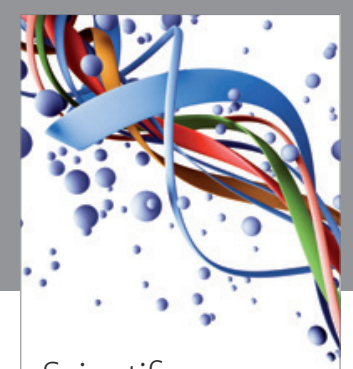

Scientifica
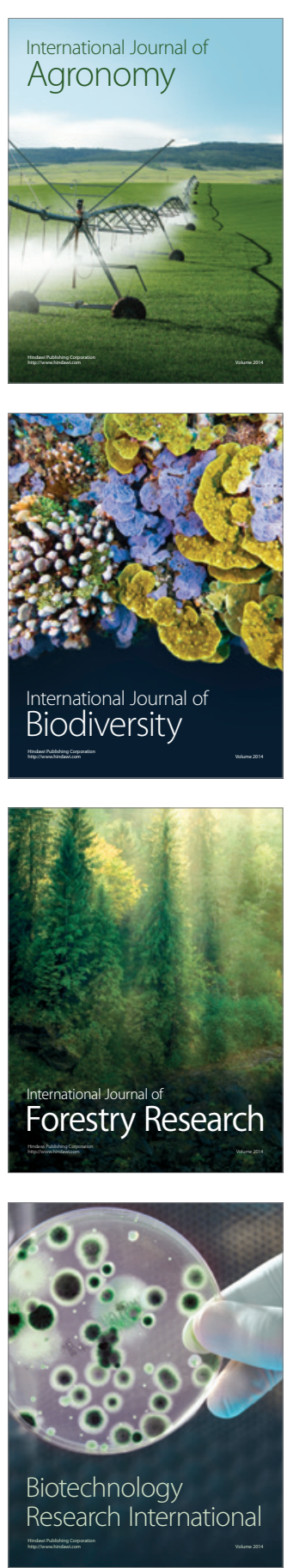
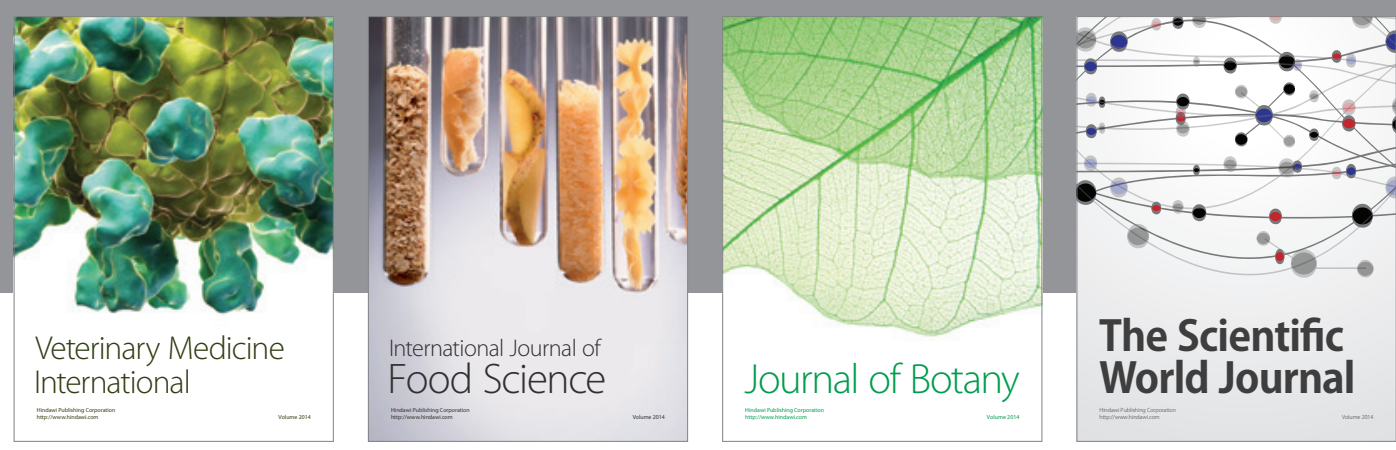

The Scientific World Journal
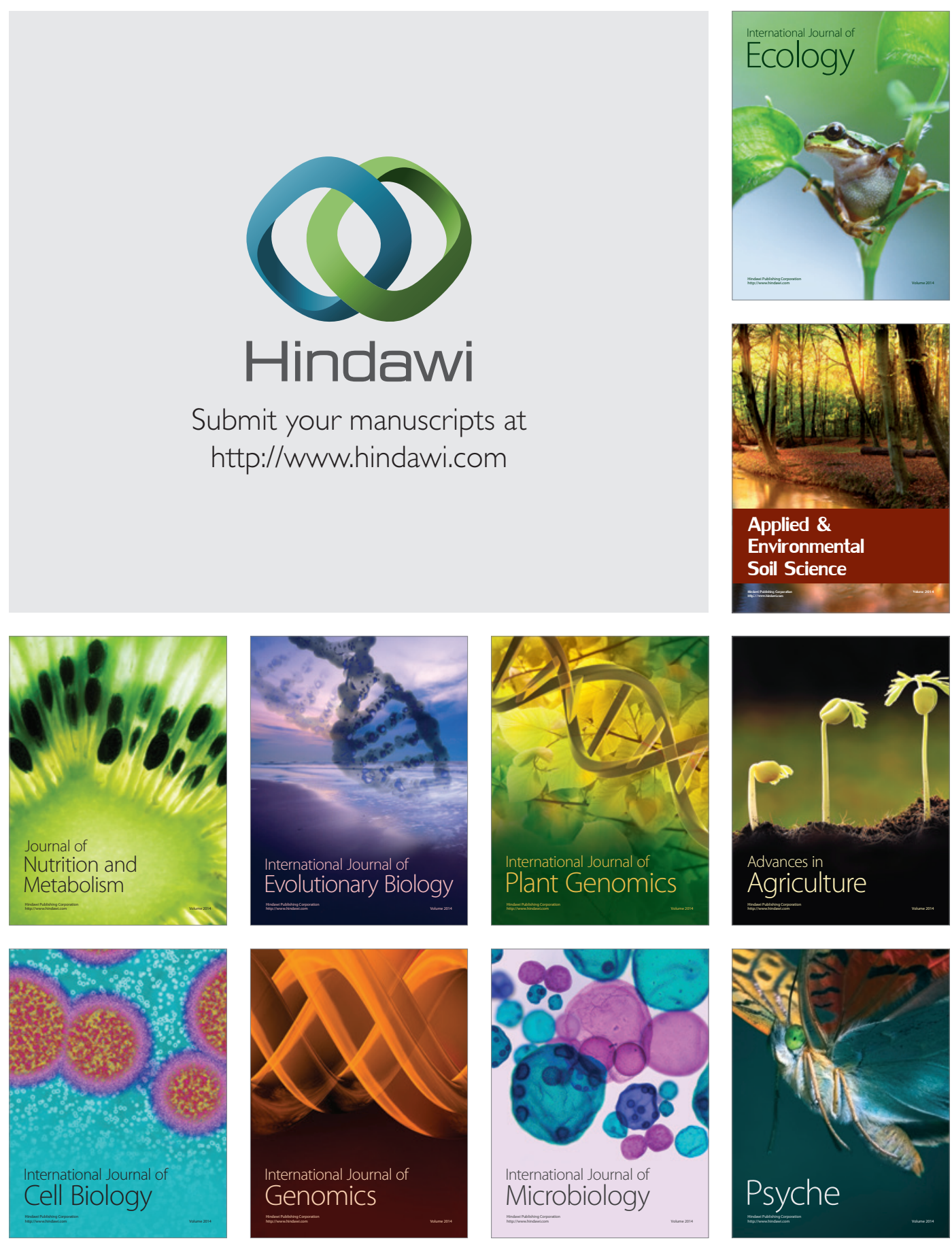